\title{
Spontaneous Splenic Rupture Following Bouts of Coughing: A Rare Case Report and Literature Review
}

\author{
Saptarshi Biswas ${ }^{\mathrm{a}, \mathrm{b}}$, Laryssa Richards ${ }^{\mathrm{a}}$
}

\begin{abstract}
Splenic rupture due to trauma is relatively common. However, spontaneous non-traumatic ruptures do occur. Causes include infection, neoplasia and infiltrative process. We present an unique case of a 59-year-old patient who presented with dyspnea and left upper abdominal discomfort following bouts of coughing, and was provisionally diagnosed as pulmonary embolism. CT scan revealed splenic rupture. Only a few case reports are published documenting spontaneous splenic rupture following coughing. The therapy of choice can vary between patients depending on the grade of splenic rupture, hemodynamic instability, availability of endovascular treatment and physician preference. Treatment should be focused on preserving splenic tissue if feasible. Non-traumatic rupture of the spleen must be considered in patients presenting with left-sided upper abdominal pain even without evident history of trauma, since early recognition and treatment can prevent serious morbidity and mortality.
\end{abstract}

Keywords: Spontaneous; Splenic rupture; Coughing

\section{Introduction}

Rupture of the spleen secondary to trauma is a potentially lifethreatening yet relatively common complication. Although non-traumatic rupture is rare, some causes include neoplastic (lymphoma and leukemia), inflammatory, and infections (Epstein-Barr virus (EBV), cytomegalovirus (CMV), hepatitis, salmonella, and malaria) [1].

Spontaneous rupture of the spleen is a condition in which the spleen ruptures in the absence of trauma or disease. Delayed or missed diagnosis can often result in significant morbidity and mortality. Overall mortality rate is reported as 10 $15 \%$ [2]. Ninety-three percent of the so-called "spontaneous

Manuscript accepted for publication November 01, 2016

aDepartment of Trauma and Acute Care Surgery, Forbes Regional Hospital, Allegheny Health Network, Pittsburgh, PA, USA

${ }^{b}$ Corresponding Author: Saptarshi Biswas, Department of Trauma and Acute Care Surgery, Forbes Regional Hospital, Allegheny Health Network, Pittsburgh, PA, USA. Email: saptarshibiswas@comcast.net

doi: https://doi.org/10.14740/jcs308w splenic ruptures" are associated with histopathologically altered spleens [2].

Spontaneous splenic rupture has been reported after trivial insults such as vomiting and coughing. We report a case of spontaneous rupture of a normal spleen after "coughing fits".

\section{Case Report}

A 59-year-old female presented to the emergency department (ED) with the chief complaint of chest pain that had started a couple of days back. It was diffusely lower. She also complained of "cough".

The patient stated that she woke up with a "coughing fit", and had felt a sharp, non-radiating pain in the left upper quadrant that got worse with breathing. The patient denied any traumatic events, shortness of breath, chest pain, headaches, fever, and recent illness. She denied history of splenomegaly.

Vitals on presentation showed temperature of $36.5^{\circ} \mathrm{C}$, heart rate of 77 , blood pressure of $107 / 73 \mathrm{~mm} \mathrm{Hg}$, respiratory rate 20 , and $\mathrm{O}_{2}$ saturation of $97 \%$.

On physical exam, the patient appeared well nourished, alert and oriented although uncomfortable. Lungs were clear to auscultation bilaterally without any obvious respiratory distress. Heart had a regular rate and rhythm. The patient's abdomen was soft and grossly non-tender except left upper quadrant (LUQ) on deep palpation, without any palpable masses, rebound or guarding. She had positive bowel sounds in all four quadrants. Rest of system review showed no gross abnormality.

Her past medical history was significant for arthritis and hypertension. Her past surgical history included appendectomy, tonsillectomy, adenoidectomy, and varicose vein surgery. Medications included aspirin and hydrochlorothiazide. The patient denied any allergies. Social history included being a former smoker.

Initial labs showed white blood cell (WBC) of $9.13 \times 10^{3 /}$ $\mu \mathrm{L}$, hemoglobin of $12.7 \mathrm{~g} / \mathrm{dL}$, hematocrit of $39.2 \%$, platelets of $220 \times 10^{3} / \mu \mathrm{L}, \mathrm{Na}$ of $141 \mathrm{mEq} / \mathrm{L}, \mathrm{K}$ of $2.5 \mathrm{mEq} / \mathrm{L}, \mathrm{Cl}$ of $95 \mathrm{mEq} / \mathrm{L}, \mathrm{CO}_{2}$ of $28 \mathrm{mEq} / \mathrm{L}$, blood urea nitrogen of $24 \mathrm{mg} /$ $\mathrm{dL}$, creatinine of $0.77 \mathrm{mEq} / \mathrm{L}$, alanine aminotransferase (ALT) of $39 \mathrm{IU} / \mathrm{L}$, aspartate aminotransferase (AST) of $33 \mathrm{IU} / \mathrm{L}$, and alkaline phosphatase (ALP) of $69 \mathrm{IU} / \mathrm{L}$. Troponins were negative. Blood gases showed a $\mathrm{pH}$ of $6.8, \mathrm{PaCO}_{2}$ of $56 \mathrm{~mm} \mathrm{Hg}$, and $\mathrm{HCO}_{3}$ of $9.3 \mathrm{mmol} / \mathrm{L}$.

A 12-lead electrocardiography was performed which 


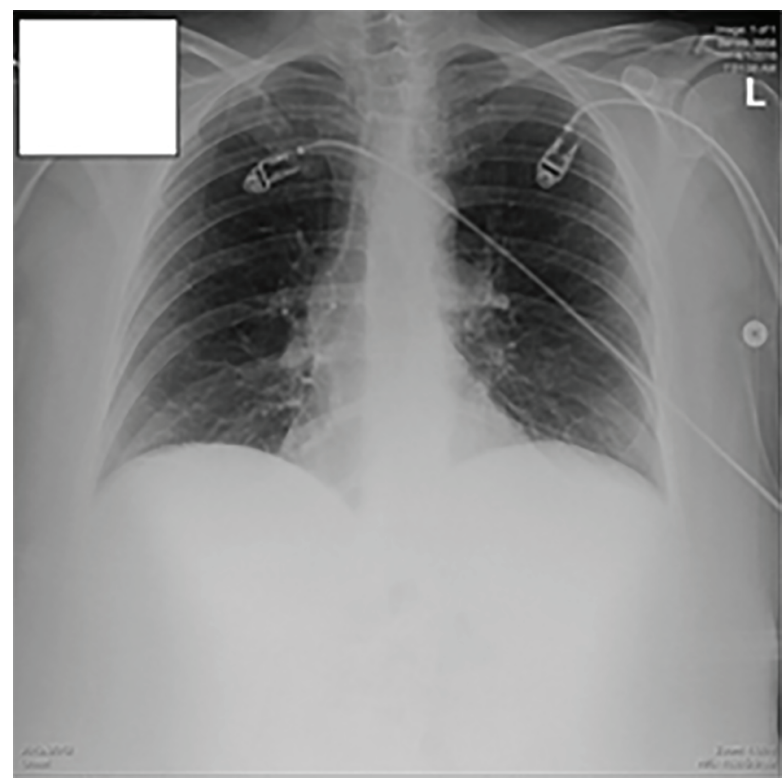

Figure 1. Chest X-ray in the ED.

showed sinus rhythm without any gross acute changes. A chest X-ray along with a flat film of the abdomen was done which was negative for any obvious pneumothorax or pneumonia (Figs. 1, 2). The provisional diagnosis was pulmonary embolism (PE).

CT scans of the chest and abdomen (Fig. 3) were conducted. Her workup for pulmonary embolus was negative; the heart was normal in size and the descending aorta and main pulmonary artery were not enlarged; however, there was presence of centrilobular emphysema and mucus plugging in both lower lobes. CT showed incidental finding of a large heterogenous perisplenic collection consistent with a hematoma and

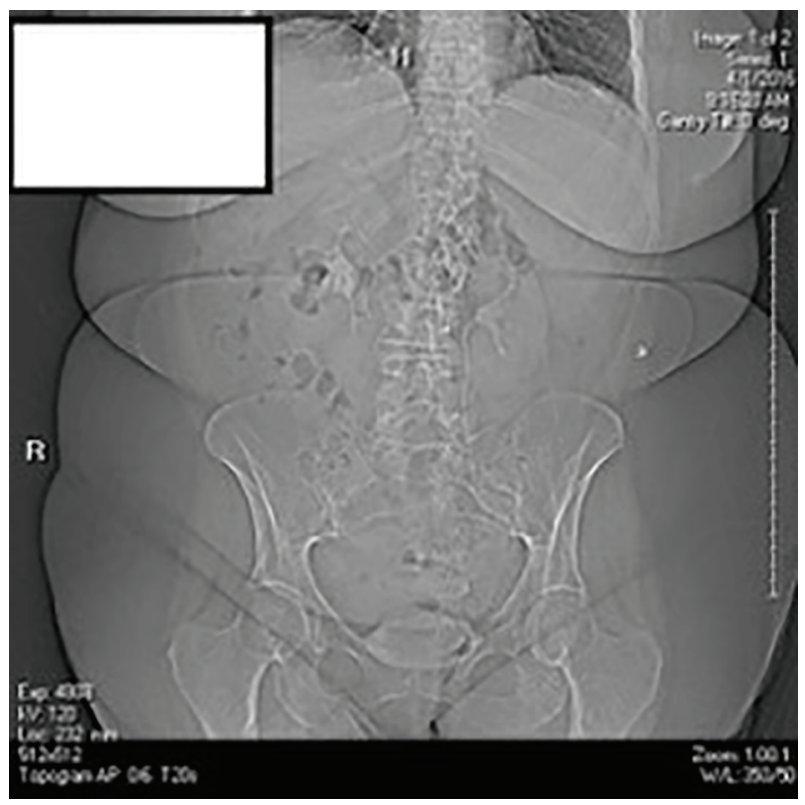

Figure 2. Abdominal X-ray in the ED.

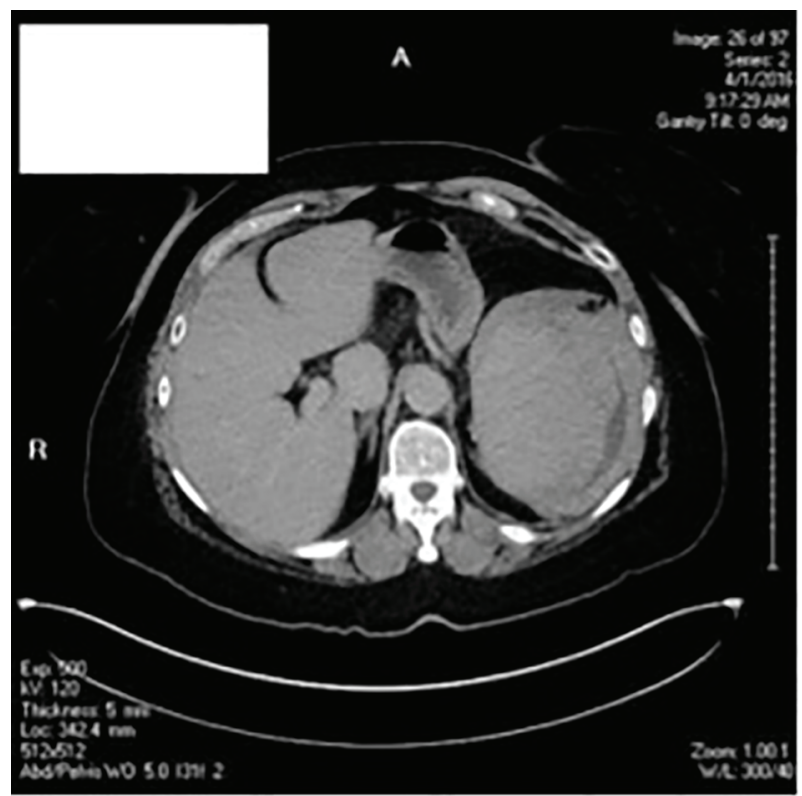

Figure 3. CT scans of chest, abdomen and pelvis.

hemoperitoneum layering in the pelvis. Acute care surgery was consulted.

The patient was admitted to the surgery floor and started on conservative management. Infectious Disease and Hematology-Oncology were consulted in order to rule out other causes of splenomegaly including EBV, CMV, and hepatitis viruses. Peripheral blood for any lymphoproliferative or leukemic cells was negative. She was also screened for JAK-2 mutation which was negative. Quantitative RT-PCR assay was negative for the b2a2 and b3a2(p210) and ela2(p190) fusion gene transcripts found in chronic myelogenous leukemia (CML) and Philadelphia-positive acute lymphocytic leukemia (ALL). Flow cytometry study showed no immunophenotypic findings diagnostic of acute leukemia or a lymphoproliferative disorder.

The patient was started on a liquid diet on hospital day 3 but developed persistent nausea, abdominal pain, and malaise. She denied shortness of breath or chest pain.

An open splenectomy was performed. An upper midline incision was made from the xiphisternum to the umbilicus. The peritoneal cavity was entered. A Bookwalter was used for exposure. The spleen had already ruptured. A ligature was used to divide the omentum and fat and the gastrosplenic omentum. Lap pads were placed behind the spleen to elevate and medialize the spleen into the midline operative field. The surgeon's left hand was passed laterally to the spleen, bluntly dissecting the splenophrenic and splenorenal ligaments, and mobilizing the spleen anteromedially into the wound. With the spleen fully mobilized, the splenic vessels were taken between clamps and ligated. The artery was always ligated before the vein. Any remaining short gastric vessels or other attachments were divided, freeing the spleen from the abdomen. Once the spleen was out, attention was placed to accessory spleen remnants. There were isolated pockets in the splenorenal ligament which were taken out by blunt dissection. The wound bed was 
thoroughly irrigated with warm saline till the return was clear. Fibrillar and avitene local hemostatic agents were placed in the splenic bed. The abdomen was closed in layers with 1-0 maxon for fascia followed by 3-0 vicryl for subcutaneous and the skin was closed with staples. The patient tolerated the surgery well.

Pathological study showed benign spleen with congestion along with fragments of hematoma. There was no definitive morphologic or phenotypic evidence for lymphoproliferative disorder.

The patient made an uneventful recovery and was discharged home on postoperative day 7 . Three vaccines were given before she left: the pneumococcal polyvalent vaccine, hemophilus B vaccine, and meningococcal polysaccharide vaccine.

The patient presented to the ED 9 days following discharge with chest pain in her left back area that began the night before. A CT scan of the chest was performed with IV contrast, which demonstrated the presence of left-sided subsegmental pulmonary emboli with moderate left pleural effusion. The patient was started on lovenox for the pulmonary emboli and had a chest tube placed to drain the pleural effusion. She was discharged home 5 days later.

\section{Discussion}

Spontaneous rupture of the normal spleen is a very rare occurrence. It accounts for up to $1 \%$ of all splenic ruptures [3]. In a comprehensive review article published in 2012 by AubreyBassler and Sowers [4], there were only 12 reported cases of spontaneous rupture of the normal spleen after coughing in English and French literature on human subjects between 1950 and 2011 [5-16].

For non-traumatic rupture of the spleen, the most common etiologies are infections, neoplasia and connective tissue disease [1]. The common causes of infiltrative or inflammatory pathology are malaria, mononucleosis, CMV, typhoid fever, endocarditis with splenic embolism, pneumonia, viral hepatitis, etc. The common hematological causes are non-Hodgkin and Hodgkin's lymphoma, ALL, CML, acute myelogenous leukemia (AML), hairy cell leukemia and histiocytosis. Some of the commonly attributed rheumatologic causes are Wegeners granulomatosis, polyarteritis nodosa, systemic lupus erythematosus, amyloidosis, etc. There have been also 38 cases of pregnancy-related rupture of normal spleen reported. There have also been previously undiagnosed non-hematological neoplastic causes of spontaneous rupture of spleen reported; the common causes cited are angiosarcoma, choriocarcinoma, pancreatic cancer, lung cancer, etc. Spontaneous splenic ruptures are also caused by anticoagulation, thrombolytics, recombinant granulocyte-colony stimulating factor, and antiplatelet agents [4].

The review article published by Aubrey-Bassler and Sowers [4] mentioned four case reports of spontaneous rupture of spleen following vomiting [1, 17-29], and one case report following seizure [20]. There have also been case reports of spontaneous rupture of spleen following colonoscopy, endoscopic retrograde cholangiopancreatography, hepatic and pulmonary surgery and even emission computed tomography, laparoscopy and hysterectomy [4].

A case of spontaneous splenic rupture was first described in 1874 by Atkinson, a British surgeon [21]. His publication triggered a number of similar case report publications eventually leading to the comprehensive review article in 1958 [22]. Orloff and Peskin recognized four-step criteria for the diagnosis of spontaneous rupture consisting of no trauma history, no perisplenic adhesions that may support previous trauma, no disease affecting the spleen, and presence of a normal spleen on gross and histologic examinations [22]. In the same review article, they cited 71 cases termed as spontaneous splenic rupture of which only 20 met all of the criteria previously mentioned. Crate and Payne added a fifth criterion: Full virological studies of acute phase and convalescent sera should show no significant rise in antibody titers suggesting recent viral infection of types known to be associated with splenic involvement [23].

The clinical picture of traumatic rupture and non-traumatic rupture of the spleen are identical. The spleen is normal on gross and microscopic examination with rupture and bleeding. The common clinical presentation is hypovolemic shock along with signs of peritonitis $[13,24]$. Upper left abdominal pain was caused by diaphragmatic irritation causing referred pain to the left shoulder (Kehr sign) and Balance sign (palpable painful mass may be felt in the LUQ). Other associated clinical findings may include nausea, vomiting, dizziness, or syncope.

When a splenic rupture is suggested, ultrasound may be used as the first step in imaging studies. Focused Assessment with Sonography for Trauma (FAST) exam is easily performed in the ED, safe, takes shorter time than CT scan and shows presence of peritoneal fluid in the LUQ. It is now considered the best initial imaging method for unstable patients [25]. Besides being operator-dependent, the FAST sometimes fails to detect fluid volumes less than 200 - $500 \mathrm{~mL}$. Plain film abdomen may show enlargement of the spleen along with medial displacement of the fundic bubble. There may be elevation of left diaphragm associated with impaired motion [26]. CT scan is presently the imaging tool of choice and shows the splenic hematoma or rupture. It has a high sensitivity and specificity in the $95 \%$ range in detecting splenic injury [27].

$\mathrm{CT}$ is important in staging and deciding if surgery is necessary [3]. Splenic injury is graded using the standards for Organ Injury Scaling Committee for the American Association of the Surgery of Trauma (AAST). Although this is developed for essentially traumatic splenic rupture, it is prudent to use for all splenic ruptures. Lower-grade injuries are typically managed conservatively while higher-grade injuries often require surgery. Nevertheless, much evidence supports the idea that the clinical circumstances should be the most important aspect in managing the patient [28].

Splenectomy remains the choice of treatment in patients presenting in hemorrhagic shock and hemodynamically unstable patients. During laparotomy repair of the spleen or absorbable mesh encasement (splenorrhaphy) to preserve splenic functions are options depending on the severity and hemodynamic status $[25,29,30]$. Transcatheter embolization is a viable alternative to surgery especially when the patients respond to volume resuscitation and remain stable. Selective 
embolization can potentially retain splenic function due to the development of collaterals [31-33]. Preservation of viable splenic tissue is desirable if possible [34]. Post-splenectomy sepsis though rare can be potentially lethal with a mortality rate ranging between $40 \%$ and $50 \%$ [34].

Hemodynamically stable patients can be observed in a monitored setting with serial abdominal exam and labs, bed rest and administration of fluid and blood as required. Rupture usually occurs during the first week although delayed rupture cases are well documented [35]. Stephenson and DuBois showed in a recent report that patients requiring less than $4 \mathrm{U}$ or $40 \mathrm{~mL} / \mathrm{kg}$ of PRBC for resuscitation can be managed conservatively [36]. However, each patient should be assessed on an individual basis with low threshold for surgical intervention in the context of any change in hemodynamic status of the patient.

In the case presented, initially, conservative management was attempted; however, this failed and the patient eventually underwent splenectomy.

\section{Conclusion}

In conclusion, many patients present to the ED with abdominal pain. The possibility of splenic rupture should be considered even in the non-traumatic patient. The delay in diagnosis can be catastrophic. Abdominal CT scan is often required to detect this condition if the clinical picture is unclear and should be done immediately.

\section{References}

1. Lemon M, Dorsch M, Street K, Cohen R, Hale P. Splenic rupture after vomiting. J R Soc Med. 2001;94(10):527528.

2. Renzulli P, Hostettler A, Schoepfer AM, Gloor B, Candinas D. Systematic review of atraumatic splenic rupture. Br J Surg. 2009;96(10):1114-1121.

3. Y Ayhan ACAR. Spontaneous Rupture of Spleen as a Rare Cause of Abdominal Pain: Case Report. European Journal of Surgical Sciences. 2010;1:27-29.

4. Aubrey-Bassler FK, Sowers N. 613 cases of splenic rupture without risk factors or previously diagnosed disease: a systematic review. BMC Emerg Med. 2012;12:11.

5. Shivashankar GH, Kelly JF. Spontaneous splenic rupture. Surgery On-line. 2007.

6. Amonkar SJ, Kumar EN. Spontaneous rupture of the spleen: three case reports and causative processes for the radiologist to consider. Br J Radiol. 2009;82(978):e111113.

7. Buciuto R, Kald A, Borch K. Spontaneous rupture of the spleen. Eur J Surg. 1992;158(2):129-130.

8. Morrin FJ, Guiney E. Spontaneous rupture of the normal spleen. Ir J Med Sci. 1961;431:500-505.

9. Rice JP, Sutter CM. Spontaneous splenic rupture in an active duty Marine upon return from Iraq: a case report. J Med Case Rep. 2010;4:353.

10. Arnold RE, Van Vooren A. Spontaneous rupture of the spleen with EMATOMA. South Med J. 1975;68(7):863864.

11. Grech A. Spontaneous rupture of spleen. Br Med J. 1971;1(5740):111.

12. Lennard TW, Burgess P. Vomiting and 'spontaneous' rupture of the spleen. Br J Clin Pract. 1985;39(10):407, 410.

13. Toubia NT, Tawk MM, Potts RM, Kinasewitz GT. Cough and spontaneous rupture of a normal spleen. Chest. 2005;128(3):1884-1886.

14. Wehbe E, Raffi S, Osborne D. Spontaneous splenic rupture precipitated by cough: a case report and a review of the literature. Scand J Gastroenterol. 2008;43(5):634-637.

15. Wergowske GL, Carmody TJ. Splenic rupture from coughing. Arch Surg. 1983;118(10):1227.

16. Kara E, Kaya Y, Zeybek R, Coskun T, Yavuz C. A case of diaphragmatic rupture complicated with laceration of stomach and spleen caused by a violent cough presenting with mediastinal shift. Ann Acad Med Singapore. 2004;33(649):650.

17. Thomas WE. Apparent spontaneous rupture of the spleen. Br Med J. 1978;1(6110):409-410.

18. Lorimer WS, Jr. Occult Rupture of the Spleen. Arch Surg. 1964;89:434-440.

19. Vas W, Friend WD. Rupture of a normal spleen caused by vomiting following a metrizamide myelogram. Diagn Imaging. 1981;50(6):309-312.

20. Drapanas T, Yates AJ, Brickman R, Wholey M. The syndrome of occult rupture of the spleen. Arch Surg. 1969;99(3):298-306.

21. Atkinson E. Death from an idiopathic rupture of the spleen. BMJ. 1874;2:403-404.

22. Orloff MJ, Peskin GW. Spontaneous rupture of the normal spleen; a surgical enigma. Int Abstr Surg. 1958;106(1):111.

23. Crate ID, Payne MJ. Is the diagnosis of spontaneous rupture of a normal spleen valid? J R Army Med Corps. 1991;137(1):50-51.

24. Debnath D, Valerio D. Atraumatic rupture of the spleen in adults. Journal of the Royal College of Surgeons of Edinburgh. 2002;47(1):437-445.

25. Michiel Leijnen, Wobbe O. de Steur, Wouter Brekelmans, Andy da Costa. Non-traumatic Rupture of the Spleen: An Atypical Presentation of the Acute Abdomen. Abdominal Surgery; Winter 2011 / Spring 2012 issue.

26. Love L, Greenfield GB, Braun TW, Moncada R, Freeark RJ, Baker RJ. Arteriography of splenic trauma. Radiology. 1968;91(1):96-102.

27. Jeffrey RB, Laing FC, Federle MP, Goodman PC. Computed tomography of splenic trauma. Radiology. 1981;141(3):729-732.

28. Becker CD, Spring P, Glattli A, Schweizer W. Blunt splenic trauma in adults: can CT findings be used to determine the need for surgery? AJR Am J Roentgenol. 1994;162(2):343-347.

29. Hamel CT, Blum J, Harder F, Kocher T. Nonoperative treatment of splenic rupture in malaria tropica: review of literature and case report. Acta Trop. 2002;82(1):1-5.

30. Leemans R, van Mourik JB. [A spleen-preserving method in splenic rupture using an absorbable net]. Ned Tijdschr 
Geneeskd. 1988;132(44):2016-2017.

31. Bellingham GA, Kribs S, Kornecki A, Scott L, Leaker M, Fraser DD. Proximal splenic artery embolization in the management of splenic rupture. Pediatr Crit Care Med. 2009;10(1):e1-4.

32. Loffroy R, Guiu B, Cercueil JP, Lepage C, Cheynel N, Steinmetz E, Ricolfi F, et al. Transcatheter arterial embolization of splenic artery aneurysms and pseudoaneurysms: short- and long-term results. Ann Vasc Surg. 2008;22(5):618-626.

33. Lagana D, Carrafiello G, Mangini M, Fontana F, Dizonno M, Castelli P, Fugazzola C. Endovascular treatment of splenic artery aneurysms. Radiol Med. 2005;110(1-2):7787.

34. Bisharat N, Omari H, Lavi I, Raz R. Risk of infection and death among post-splenectomy patients. J Infect. 2001;43(3):182-186.

35. Julia J, Canet JJ, Lacasa XM, Gonzalez G, Garau J. Spontaneous spleen rupture during typhoid fever. Int J Infect Dis. 2000;4(2):108-109.

36. Stephenson JT, DuBois JJ. Nonoperative management of spontaneous splenic rupture in infectious mononucleosis: a case report and review of the literature. Pediatrics. 2007;120(2):e432-435. 